Supporting Information

\title{
MSLDOCK: Multi-Swarm Optimization for Flexible Ligand Docking and Virtual Screening
}

\author{
Chao $\mathrm{Li}^{1}$, Jun Sun*1, Vasile Palade ${ }^{2}$ \\ ${ }^{1}$ Department of Computer Science and Technology, Jiangnan University, No.1800, Lihu Avenue, \\ Wuxi, Jiangsu 214122, PR China \\ ${ }^{2}$ Faculty of Engineering and Computing, Coventry University, Priory Street, Coventry, CV1 5FB, \\ UK
}

*Email: junsun@jiangnan.edu.cn 


\section{Search Performance Comparison on MSLDOCK Versions with Different Number of Sub-Swarms}

We employed this set of experiments in order to analyze how the number of subswarms affects the search performance of MSLRDPSO algorithm, and then we can preliminarily determine the selection of parameter $T$ (number of sub-swarms). Therefore, the performance metric used in this experiment is the docked energy, which is the energy value calculated by the semi-empirical scoring function in Autodock 4.2.6. The docked energy directly reflects the search performance of the algorithms. It includes the intermolecular and intramolecular interaction energies.

In this set of experiments, a collection of total 388 test cases in two datasets was used, including 285 test cases in PDBbind coreset and 103 test cases in GOLD dataset. The descriptions of these two datasets can be found in the main text. With respect to the parameter settings, the number of particles was set to 150 , the number of energy function evaluations were set to $2.5 \times 10^{6}$, and the maximum number of iterations of single SWLS method was set to 300 . The repetitions for each test case was set to 50. Based on the datasets and the parameter settings, the results of the docked energy obtained by the MSLDOCK with 1, 2, 4, 6, and 8 sub-swarms, i.e., MSLDOCK-s1, MSLDOCK-s2, MSLDOCK-s4, MSLDOCK-s6, and MSLDOCK-s8, were compared with each other. Note that the performance of the MSLDOCK-s1 was evaluated to show the difference between the MSLDOCK versions with single and multiple sub-swarms. 
Table S4. Comparison of the mean docked energy results among MSLDOCK versions with different number of sub-swarms

\begin{tabular}{|l|c|c|c|c|}
\hline & $\begin{array}{c}\text { Average energy } \\
\text { (Kcal/mol) }\end{array}$ & $\begin{array}{c}\text { Average standard } \\
\text { deviation }^{\mathbf{2}} \text { (Kcal/mol) }\end{array}$ & P-value-s2 $^{\mathbf{3}}$ & $\boldsymbol{P}_{\text {-value-s6 }}$-val $^{\mathbf{m}}$ \\
\hline MSLDOCK-s1 & -9.382 & 1.446 & $2.574 \mathrm{e}-65$ & $2.495 \mathrm{e}-60$ \\
\hline MSLDOCK-s2 & -10.704 & 1.078 & & $6.595 \mathrm{e}-64$ \\
\hline MSLDOCK-s4 & -11.155 & 0.780 & $1.035 \mathrm{e}-60$ & $1.095 \mathrm{e}-29$ \\
\hline MSLDOCK-s6 & -11.280 & 0.640 & $6.595 \mathrm{e}-64$ & \\
\hline MSLDOCK-s8 & -11.355 & 0.688 & $8.465 \mathrm{e}-65$ & $1.563 \mathrm{e}-13$ \\
\hline
\end{tabular}

${ }^{1}$ Average value of the mean docked energy after 50 repetitions for all test cases

${ }^{2}$ Average value of the standard deviation after 50 repetitions for all test cases

${ }^{3} P$-value for the results obtained by the corresponding program and MSLDOCK-s2

${ }^{4} P$-value for the results obtained by the corresponding program and MSLDOCK-s6

Table S5. Comparison of the lowest docked energy results among MSLDOCK versions with different number of sub-swarms

\begin{tabular}{|l|c|c|c|}
\hline & $\begin{array}{c}\text { Average energy } \\
\left.\mathbf{~}^{\mathbf{K c a l}} / \mathbf{m o l}\right)\end{array}$ & $\boldsymbol{P}$-value-s2 & $\boldsymbol{P}^{2}$-value-s6 \\
\hline MSLDOCK-s1 & -11.995 & $7.223 \mathrm{e}-25$ & $1.117 \mathrm{e}-22$ \\
\hline MSLDOCK-s2 & -12.195 & & 0.052 \\
\hline MSLDOCK-s4 & -12.233 & 0.619 & 0.082 \\
\hline MSLDOCK-s6 & -12.208 & 0.052 & \\
\hline MSLDOCK-s8 & -12.218 & 0.464 & 0.789 \\
\hline
\end{tabular}

${ }^{1}$ Average value of the lowest docked energy of 50 repetitions for all test cases

${ }^{2} P$-value for the results obtained by the corresponding program and MSLDOCK-s2

${ }^{3} P$-value for the results obtained by the corresponding program and MSLDOCK-s6

Table S4 and Table S5 illustrate some statistical results in terms of docked energy obtained by different MSLDOCK versions for all the 388 test cases. The $P$-values (calculated by using Wilcoxon sign-ranked test) in Table S4 are all less than 0.05 , which shows that the difference between the average values of the mean docked energy obtained by different versions of MSLDOCK are significant at 95\% confidence level. Specially, Table S4 illustrates that the value of the "Average energy" decreases with the increase of the number of sub-swarms. Moreover, the MSLDOCK with 6 sub-swarms can get the best mean standard deviations for all test cases among all the compared algorithms, 
followed by MSLDOCK-s8, and then the others MSLDOCK versions. This indicates that the MSLDOCK versions with more sub-swarms perform better robustness. The MSLDOCK versions with a few sub-swarms, such as MSLDOCK-s2, can only obtain relatively high values of "Average energy" and "Average standard deviation", since their lower frequency of feature exchange results in a relatively higher probability of premature convergence than the other versions. On the other hand, the $P$-values in Table S5 demonstrate that except MSLDOCK-s1, the average values of the lowest docked energy obtained by the other versions of MSLDOCK are equivalent.

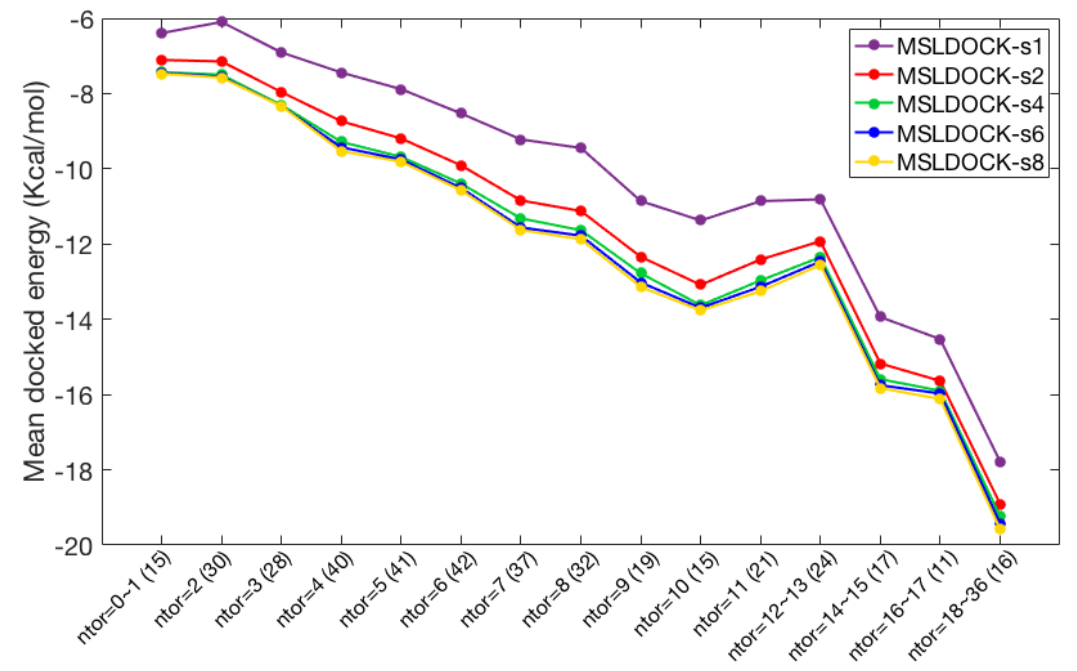

Figure S1. Mean docked energy performance in terms of torsions. For each class, every point represents the average value of the mean docked energy for all test cases in this class. 


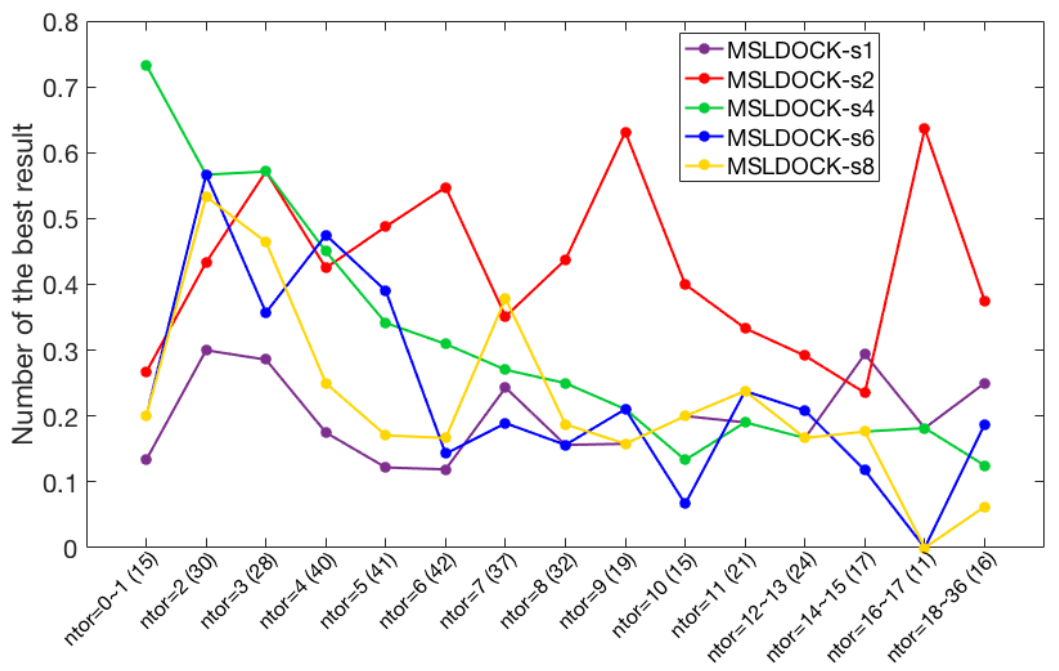

Figure S2. Lowest docked energy performance in terms of torsions. For each class, every point represents the number of the test cases for which the corresponding docking program can get the best result among the number of the test cases in this class.

In order to evaluate the search performance of the compared MSLDOCK versions for different number of search dimensions, we also illustrate the mean docked energy performance and the lowest docked energy performance of each MSLDOCK version in terms of the number of torsions in Figure S1 and Figure S2, respectively. In these two figures, the labels on the horizontal axes represent the classification of all test cases according to the number of torsions (ntor), and the value in the brackets for each label illustrates the number of the test cases in the corresponding class. According to Figure S1, for all the classes of torsions, MSLDOCK-s6 and MSLDOCK-s8 are comparable, but a little better than MSLDOCK-s4, and superior to MSLDOCK-s2 and MSLDOCKs1. With respect to Figure S2, it is obvious that MSLDOCK-s2 can get the best results in most of the classes, which illustrates the superiority of MSLDOCK-s2 in terms of the lowest docked energy. It should be noted that in some classes with less flexible ligands, other versions of MSLDOCK shows a comparable or a little better performance than 
MSLDOCK-s2. However, for the test cases with more torsions, the advantage of MSLDOCK-s2 over other MSLDOCK versions is more obvious than that for the less flexible ligand docking. The phenomena in both Figure S1 and Figure S2 verify that in terms of different number of torsions, MSLDOCK with more sub-swarms performs better mean docked energy performance, while the versions with less sub-swarms can obtain better lowest docked energy, especially for docking with highly flexible ligands.

In addition to the analysis of the final docked energy obtained by different MSLDOCK versions, we also show in Figure S3 the convergence curves of the docked energy obtained by each compared MSLDOCK version for three test cases, i.e., 1gpk, $3 \mathrm{u} 8 \mathrm{k}$ and $5 \mathrm{tmn}$. The plotted trial for each version of MSLDOCK was the one for which the corresponding program found the lowest docked energy out of 50 runs. Figure S3 illustrates that except MSLDOCK-s1, the convergence speed of the other MSLDOCK versions becomes faster in the first half of the search process with the increase of the number of sub-swarms. The reason is that for the MSLDOCK versions with more subswarms, the more frequent information exchange can make the global best value update quickly. Moreover, the higher frequency of feature exchange between different subswarms can also result in that the MSLDOCK versions with more sub-swarms have smaller standard deviations than those with less sub-swarms, and vice versa, as the results shown in Table S4. Therefore, with small standard deviations, the MSLDOCK versions with a relatively large number of sub-swarms can find enough low final docked energy in many cases. On the other hand, since the MSLDOCK versions with relatively few subswarms has large standard deviations, sometimes they have a chance to find lower 
docked energy than all the other MSLDOCK versions, but they also have opportunities to fall into local optima in the early stage of the search process. Take the performance of MSLDOCK-s2 in Figure S3 as examples: MSLDOCK-s2 can get the lowest final docked energy among all the MSLDOCK versions for 1gpk and 5tmn, but only obtain a final result much worse than those obtained by most of the other compared versions for $3 \mathrm{u} 8 \mathrm{k}$. Nevertheless, the statistical results in Figure S2 still illustrate that, if there are sufficient repetitions of a single docking test case, MSLDOCK-s2 has a relatively high opportunity to find better lowest docked energy out of multiple trials than all the other compared versions.

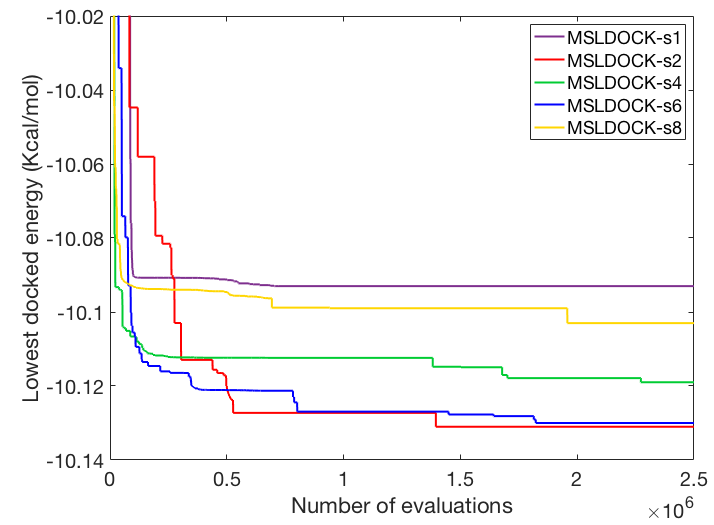

(A)

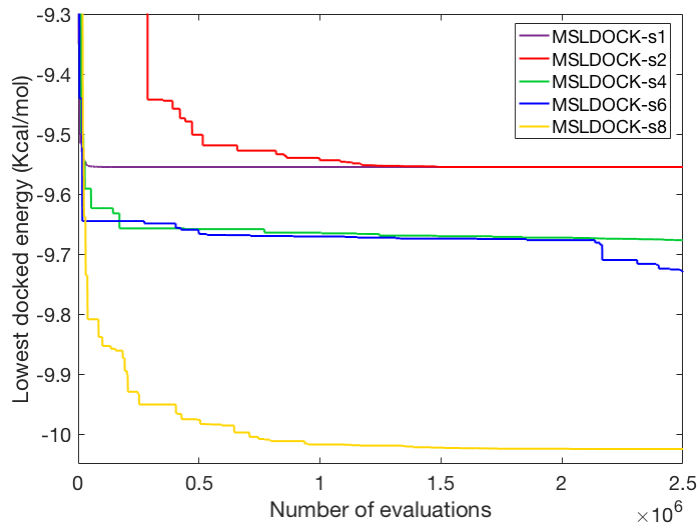

(B)

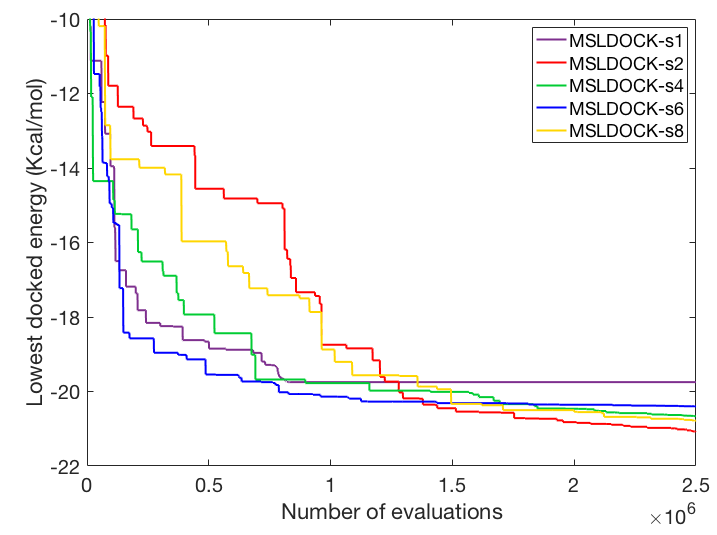

(C)

Figure S3. Convergence performance of the lowest docked energy obtained by different MSLDOCK versions during the search process for (A) 1gpk with 3 torsions, (B) 3u8k with 4 torsions and (C) 5tmn with 18 torsions. 
Based on the aforementioned analysis, it can be concluded that MSLDCOK-s1 does not has good search performance, since it obtained the worst results for almost all the evaluation criteria among all compared versions and could converge prematurely easily. With respect to the other compared versions of MSLDOCK, with the increase of the number of sub-swarms, the MSLDOCK can get lower mean docked energy and smaller standard deviation, but its performance of obtaining better lowest docked energy becomes worse if there are sufficient docking repetitions for a single test case. Therefore, MSLDOCK-s2 is considered to be the most suitable version for docking experiments with a large number of repetitions, while the MSLDOCK versions with a relatively large number of sub-swarms are more appropriate for "fast" docking, that is, docking with a small number of repetitions. As MSLDOCK-s6 has the smallest standard deviation among all compared versions and its mean docked energy performance in terms of different number of torsions can be comparable to MSLDOCK-s8, MSLDOCK with 2 and 6 sub-swarms (i.e., MSLDOCK-s2 and MSLDOCK-s6) were chosen to compared with other docking programs in further experiments in the main text. 